\title{
Acuchimay: asentamiento prehispánico de época Warpa y Wari en la cuenca de Ayacucho
}

\section{Acuchimay: pre-Hispanic settlement from the Warpa and Wari period in the Ayacucho basin}

\author{
Héctor Carhuas Tenorio \\ https://orcid.org/0000-0001-7674-3255 \\ Universidad Nacional de San Cristóbal de Huamanga \\ hectorcarhuasgo@gmail.com
}

\section{RESUMEN}

Se presenta parte de un trabajo de investigación que involucra a la arqueología de la Sierra surcentral del territorio andino, correspondiente al marco cronológico del Periodo Intermedio Temprano y Horizonte Medio. Acuchimay es uno de los asentamientos prehispánicos adyacentes al sitio arqueológico de Rudaqasa, localizado en el corazón de la actual ciudad de Ayacucho, que guarda el testimonio de una ocupación antigua de época Warpa y Wari, al igual que otros sitios, ubicados a lo largo de la cuenca del río Huarpa. El objetivo fundamental es conocer y recuperar toda la información posible existente para definir la importancia del sitio durante las épocas Warpa y Wari. El método empleado es descriptivo y síntesis a partir de la visita realizada alrededor de Acuchimay y del material cultural recuperados de la superficie; asimismo, se complementa con algunas colecciones particulares existentes en las propiedades de la población moderna del sitio. En base a las evidencias arqueológicas alcanzamos una visión general acerca de la característica del sitio para comprender sobre lo que fue la dinámica sociocultural en la antigua cuenca de Ayacucho.

Palabras clave: Acuchimay, Warpa, Wari, prehispánico, cuenca de Ayacucho. 


\section{ABSTRACT}

Part of a research work that involves the archeology of the south-central Sierra of the Andean territory is presented, corresponding to the chronological framework of the Early Intermediate Period and the Middle Horizon. Acuchimay is one of the pre-Hispanic settlements adjacent to the archaeological site of Rudaqasa, located in the heart of the current city of Ayacucho, which keeps the testimony of an ancient occupation of the Warpa and Wari times, as well as other sites, located throughout the Huarpa river basin. The fundamental objective is to know and retrieve all the possible existing information to define the importance of the site during the Warpa and Wari times. The method used is descriptive and synthesized from the visit around Acuchimay and the cultural material recovered from the surface; likewise, it is complemented by some private collections existing in the properties of the modern population of the site. Based on the archaeological evidence, we reached a general vision about the characteristic of the site to understand what the sociocultural dynamics was in the ancient Ayacucho basin.

Keywords: Acuchimay, Warpa, Wari, pre-hispanic, Ayacucho basin.

Recibido: 02/07/2021 - Aceptado: 13/08/2021 - Publicado: 25/11/2021

\section{INTRODUCCIÓN}

Acuchimay es uno de los asentamientos prehispánicos poco conocidos, que se localiza en la periferia suroeste de la ciudad Wari, en el valle de Ayacucho. Este sitio por su formación natural vistosa ha sido calificado por el tradicionista Ayacuchano Juan de Mata Peralta, como: la "Atalaya de la ciudad de Ayacucho". Además, el cerro posee un contenido mítico, religioso e histórico siendo el escenario de una de las batallas importantes de la resistencia de La Breña en el año 1882, dirigido por el Mariscal Andrés Avelino Cáceres. Además, es una imponente formación geológica, que se localiza dentro del antiguo barrio tradicional de Qarmenqa, que hoy es el actual distrito de Carmen Alto.

Actualmente Acuchimay es un mirador turístico ampliamente visitado por los excursionistas que arriban a la ciudad de Ayacucho, especialmente cada año en la mañana de sábado de gloria de la Semana Santa se realiza una de las ferias más grandes e importantes de la región de Ayacucho. La gran mayor parte de su territorio está ocupado por la población moderna y solo un pequeño espacio queda libre con afloramiento de rocas volcánicas, que contiene un abrigo rocoso sacralizado y simbólico producto de la religiosidad andina, que recibe a muchos peregrinos de la cosmovisión andina. En esta zona actualmente perdura la veneración a los Apus o Wamanis locales, que posiblemente su origen es desde los 
tiempos muy remotos y que cumplieron funciones muy importantes en el valle de Ayacucho. Según Sabino Arroyo (2006), Acuchimay es considerado como el Apu local de mayor prestigio asociado a cuatro "cruces de mayo", que se ubican orientadas con la mira a la ciudad principal, siendo uno de ellos direccionado al barrio de Qarmenqa; asimismo, la presencia del abrigo rocoso conocido como la puerta de la montaña, está vinculado a la medicina andina.

El cerro Acuchimay aparte de ser el corazón de la ciudad de Ayacucho es uno de los aposentos prehispánicos de la cultura Warpa, que fue habitado desde el periodo Intermedio Temprano (200 d. C. a 500 d. C.). De la misma forma, ha continuado durante el Horizonte Medio con el dominio Wari (500 d. C. a 1000 d. C.), un asentamiento resaltante en la periferia suroeste de la gran urbe Wari. Tanto Acuchimay como Rudaqasa, ubicado junto a este, son sitios que han pasado por una serie de cambios a lo largo de los últimos años al igual que los establecimientos cercanos como Waychaupampa, Ñawinpuquio, Conchopata, Moyo Orqo, Tunasniyuq y Aqo Wayqo; todos ubicados dentro del actual ciudad de Ayacucho, donde casi nada queda de los vestigios prehispánicos de los antiguos pobladores de este valle (figura 1).

Los estudios arqueológicos se remota a la tercera década del siglo pasado donde fue referenciado por Benedicto Flores como parte de sus labores de reportes históricos junto a los petroglifos de Ñawinpuquio. Al efectuar una serie de trabajos preparatorios y excavaciones durante más de una semana identifica hornos que lo denomina "oficinas prehistóricas de carbonizar osamentas humanas" (Lumbreras, 1974). Años después en 1942, Tello durante su expedición al Vilcamayo, realiza una serie de excavaciones en Wari y Conchopata, donde visita al cerro Acuchimay, identificando numerosos fragmentos de cerámica Wari asociados a muros de piedra que corresponden a hornos con paredes enlucidas halladas por Flores (Tello, 2014).

Años más tarde, en 1950 Bennett (1953) realiza pequeñas excavaciones tanto en Wari, Conchopata y Acuchimay, hallando arquitectura y fragmentería de cerámica Warpa y Wari. Define el estilo "Acuchimay", con su variante "Acuchimay policromo", que más tarde Lumbreras (1959), denomina como "Ayacucho Temprano", ubicado dentro de la cerámica Warpa con fuerte influencia Nasca. Los diseños resaltantes corresponden a volutas, ganchos, motivos radiales, líneas rectas perpendiculares paralelas; así como bandas quebradas en zig-zag paralelas dispuestas horizontalmente. Por un lado, a la variante "Acuchimay Polícromo", Benavides (1965), nombra como Tinajeras Zigzag, representados por cántaros y cuencos que llevan como decoración líneas zigzagueantes. Aparte de ello, Menzel (1968), lo incluyó dentro del estilo Chakipampa A, por tratarse de motivos que provienen de Warpa y la tradición Nasca.

Años más tarde, Lumbreras $(1959,1974)$ enfatiza que Acuchimay es uno de los sitios arqueológicos más conocidos en Ayacucho, perteneciente a los finales del pe- 
riodo Warpa e inicios de Wari. Además, cerca de Acuchimay, en Rudaqasa identifica abundante cerámica Wari vinculado a los primeros momentos de la presencia Tiwanaku en Ayacucho (Lumbreras 1959). Los trabajos de esta época se complementan con los reportes de Benavides (1976), que hasta ese momento señala que contaba con una serie de terrazas y muros de contención, emplazada cerca de Rudaqasa con abundante cerámica Wari de estilo Ocros y Totora.

Finalmente, López (2014) recuperó abundantes fragmentos de cerámica de época Warpa de los estilos Warpa Negro sobre Blanco, Warpa Negro sobre Rojo y Warpa Tricolor; asimismo cerámica Wari de los estilos Ocros, Chakipampa, Viñaque y Huamanga. Indica la presencia de restos de recintos circulares, semicirculares y rectangulares asociados a varios entierros humanos.

Es evidente el actual estado situacional de Acuchimay, que por su ubicación dentro de la población moderna se ha llevado al riesgo de alteración y el proceso de desaparición de numerosos testimonios de la cultura material, siendo causa principal el crecimiento demográfico poblacional. Existen muy poca información recuperada mientras la mayor parte de su área han desaparecido en su totalidad como muchos sitios arqueológicos con la construcción de viviendas y obras públicas de los últimos años. Las referencias conducen a cuestionar el rol que cumplió el asentamiento Acuchimay durante la época Warpa y Wari en el valle de Ayacucho y como estuvo el vínculo con los asentamientos circundantes. Esta interrogante condujo a recorrer las actuales calles de Acuchimay y acceder a colecciones particulares, con el objetivo de comprender el antiguo poblado prehispánico del valle de Ayacucho.

\section{UBICACIÓN DEL SITIO}

Se encuentra ubicado en el corazón de la actual ciudad de Ayacucho, en la jurisdicción de los distritos de Carmen Alto y San Juan Bautista, provincia Huamanga, región Ayacucho. Geográficamente se localiza en la cuenca media del río Huarpa, en la margen derecha del río Alameda, sobre una colina cono truncada, en las coordenadas UTM: 18L 584388E, 8543670N, a una altitud de 2849 m s. n. m. Geológicamente se encuentra constituido por rocas volcánicas de origen Terciario - Cuaternario, con abundante presencia de roca conglomerados, arenisca de familia andesita y riolita (Pérez et al., 2010). El relieve es de suave pendiente, dañada por la erosión pluvial y por el avance demográfico de la población actual quedando semiárida con escasa vegetación de la zona. Lo que resalta son vegetaciones típicas del piso ecológico quechua (Pulgar, 1996) como el molle (Schinus molle), una de las plantas nativas que ampliamente utilizamos desde las épocas muy antiguas para la elaboración de chicha y el teñido de los textiles. Asimismo, abundan la tuna (Opuntia ficus-indica), cabulla (Agave americana), ataqu (Amaranthus hybridus), wakatay (Tegetes minuta) y gramíneas típicas de este piso altitudinal. 


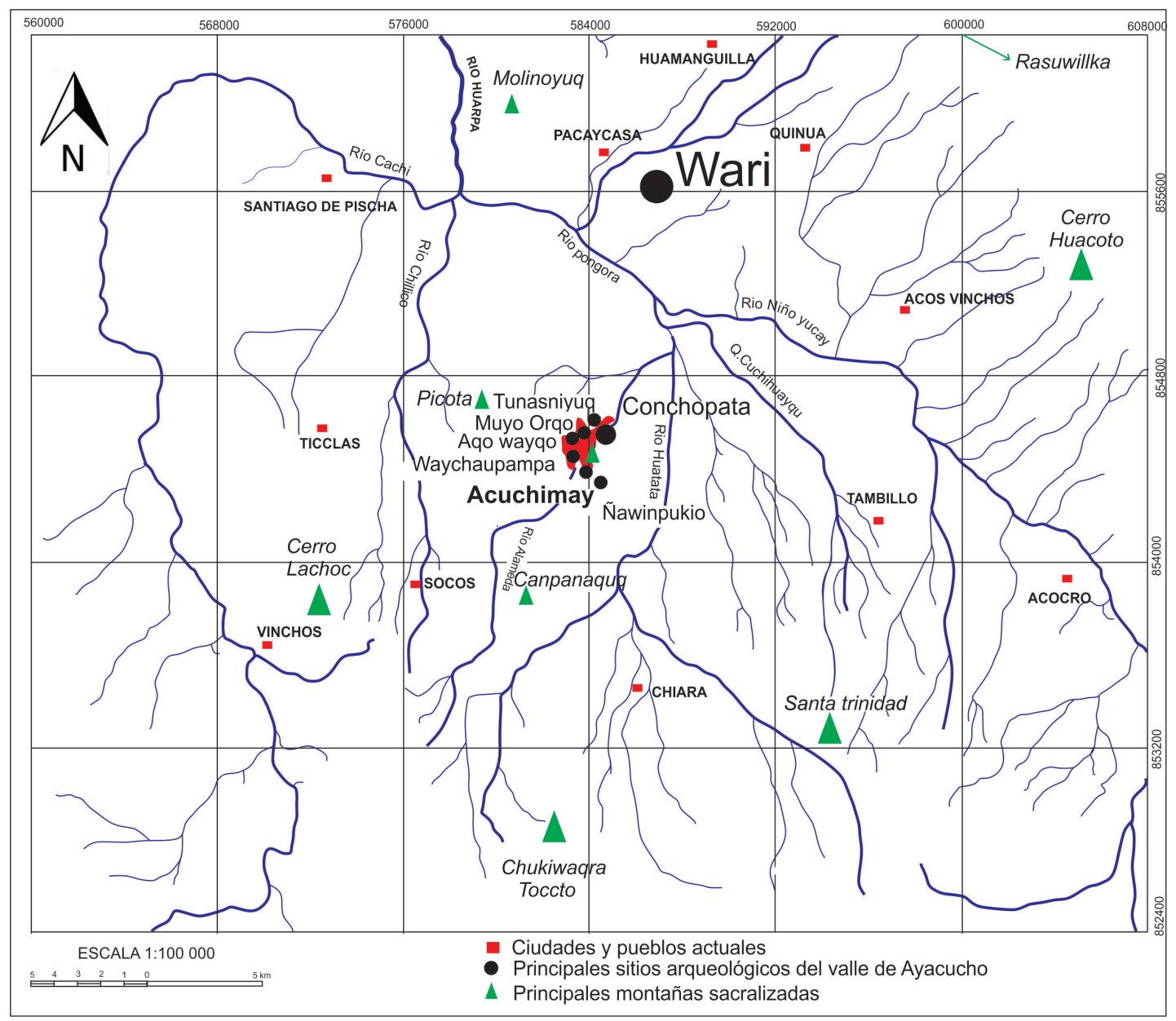

Figura 1. Ubicación de Acuchimay y los sitios adyacentes en la cuenca de Ayacucho.

\section{METODOLOGÍA DEL REGISTRO}

El desarrollo del trabajo se desarrolló con el acopio de una serie de informaciones arqueológicas con el trabajo de campo en Acuchimay y los alrededores en el distrito de Carmen Alto, en la actual ciudad de Ayacucho. Se ha contado con algunos planos, fotografías satelitales, GPS referencial y cámara fotográfica. La salida se realizó en diferentes oportunidades de manera directa con un recorrido por los diferentes sectores de Acuchimay y Rudaqasa, con la visita a propiedades particulares de algunas pobladoras de la zona, quienes de manera muy gentil accedieron sus colecciones particulares, para realizar las respectivos fotografías y dibujos.

De la misma forma, conseguimos vislumbrar abundante material arqueológico incrustado en las paredes de las viviendas y murallas perimétricas, como parte de un 
testimonio cultural prehispánico de una población que habitó hace muchos años en el valle de Ayacucho. A esto se complementa el trabajo de gabinete con la identificación del material arqueológico, determinación de la secuencia cultural y la contextualización de toda la información.

\section{IMPORTANCIA DE ACUCHIMAY DURANTE LA ÉPOCA WARPA Y WARI}

En la arqueología andina hay muchos procesos históricos desde que el hombre ha arribado al continente americano. Una parte de estos sucesos culturales en el área de los Andes Centrales, tras el ocaso de una de las sociedades teocráticas como fue Chavín de Huántar, ocurre un cambio sociopolítico y cultural de nuevos grupos y la primera diferenciación local con un nuevo tipo de organización de carácter regional (Matos, 1981). Este lapso de tiempo también es conocido como el Periodo Intermedio Temprano o Desarrollos Regionales, que, en el valle de Ayacucho, está representado por la cultura Warpa, que se desarrolló alrededor de los años 100 d. C. a $500 \mathrm{~d}$. C., previo al surgimiento del imperio Wari. Esta sociedad local ha ocupado los valles de Ayacucho, Pampas, Apurímac, la parte baja del río Mantaro y asimismo existe una serie de asentamientos en el valle de Huanta (Valdez, 2003). Esta cultura tuvo un desarrollo tecnológico fabuloso en el campo de la agricultura, con la construcción de numerosas terrazas, canales de irrigación y reservorios que mantenía exitosamente las áreas agrícolas (Ochatoma y Cabrera, 2010), así como también el dominio la parte alta para adquirir recursos naturales (Carhuas, 2020). Además, esta sociedad local ha sido la base principal de una interacción con las culturas de la Costa Sur, como resultado de ello en el valle de Ayacucho, se ha demostrado el contacto desde el Formativo Tardío, en la cerámica Chupas y Rancha (Lumbreras, 1974). También se manifiesta en los alfares de época Warpa; asimismo con mayor énfasis en la cerámica Wari de estilo Chakipampa a la cual influenció directamente.

Parte de los trabajos arqueológicos del siglo pasado se han atribuido a Ñawinpukio, como posible capital de los Warpa, ubicado al sureste de la ciudad de Ayacucho (Lumbreras, 1974). Este paradigma en los últimos años se viene remplazando con los nuevos hallazgos en la ciudad de Huari, una superposición de arquitectura temprana que define a la posible sede o capital de los Warpa (Ochatoma et al, 2015). Durante este periodo, tanto Acuchimay como Rudaqasa, no estaban ajenos a la organización sociocultural y agrícola de los Warpas, siendo Acuchimay un asentamiento habitacional con un patrón de recintos circulares emplazados en la ladera del cerro Acuchimay (Pérez, 2013). Además, es posible que en los alrededores existían numerosas terrazas agrícolas y canales hidráulicos de los que en la actualidad ya no queda ningún vestigio arqueológico.

Los estudios arqueológicos revelan que Acuchimay, fue un lugar de prestigio durante el Intermedio Temprano, habitado por una población Warpa, cuyos materia- 

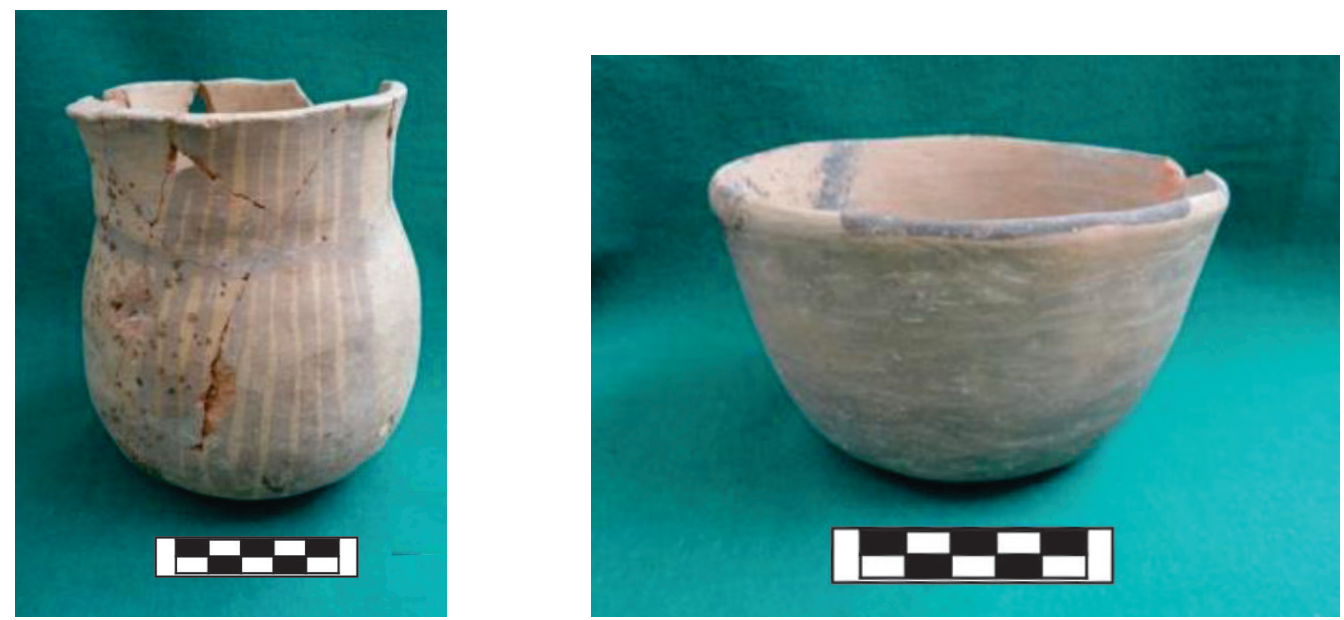

Figura 2. Detalle de cerámica Warpa Negro sobre Ante, procedentes del sitio Acuchimay (Pérez, 2016, p. 216).

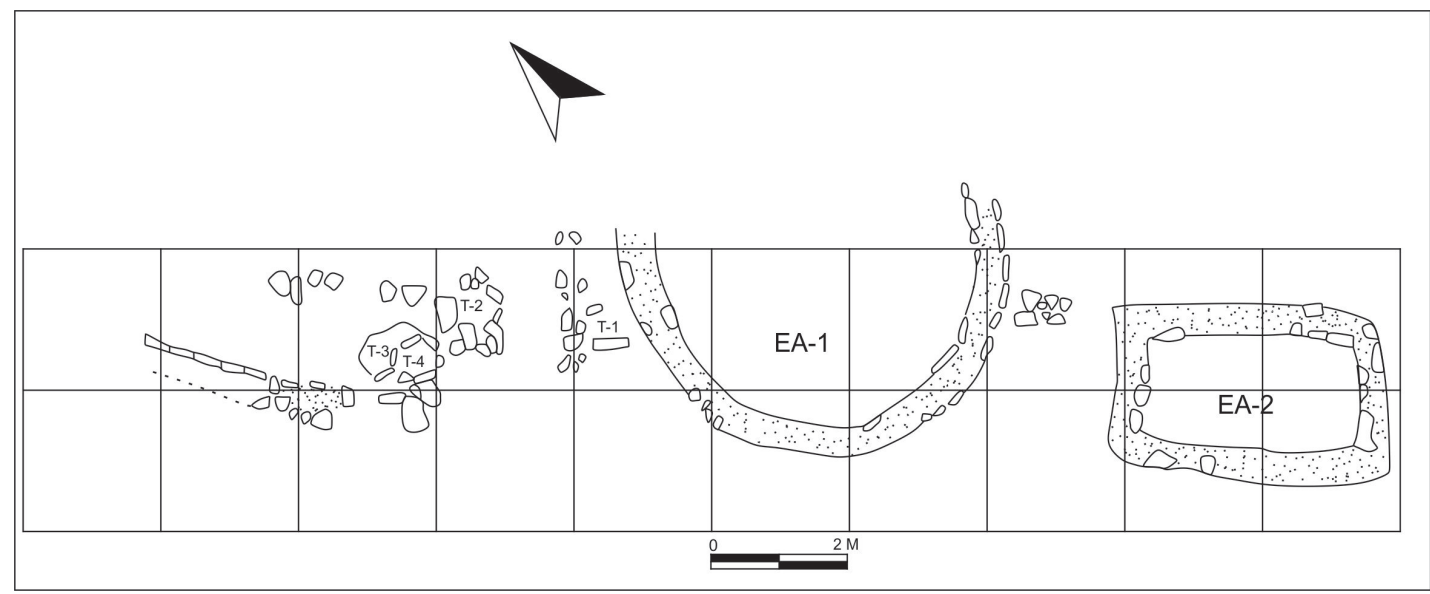

Figura 3. Dibujo en planta del recinto circular y rectangular expuesta durante el rescate arqueológico en Acuchimay (López, 2014, p. 78).

les hallados durante el recorrido de campo, fueron en su mayoría cerámicas de los estilos Kumunsenqa, Warpa Ante, Warpa Negro sobre Ante, Warpa Rojo sobre Ante, Warpa Negro sobre Blanco y Warpa Polícromo (figura 7, 8, 9). De igual forma, los reportes existentes (Benavides, 1976 y López, 2014) han mencionado la recurrencia de tipos de alfares cuyas características permiten asociar cronológicamente al segundo momento de las tres etapas propuestas para la cerámica procedente de Ñawinpuquio (Ochatoma y Cabrera, 2010). La segunda fase está vinculada con la etapa propiamente Warpa, donde los diseños son geométricos y se da el uso de pocos colores como por ejemplo en los estilos Warpa Ante, Warpa Negro sobre Ante, Warpa Ne- 


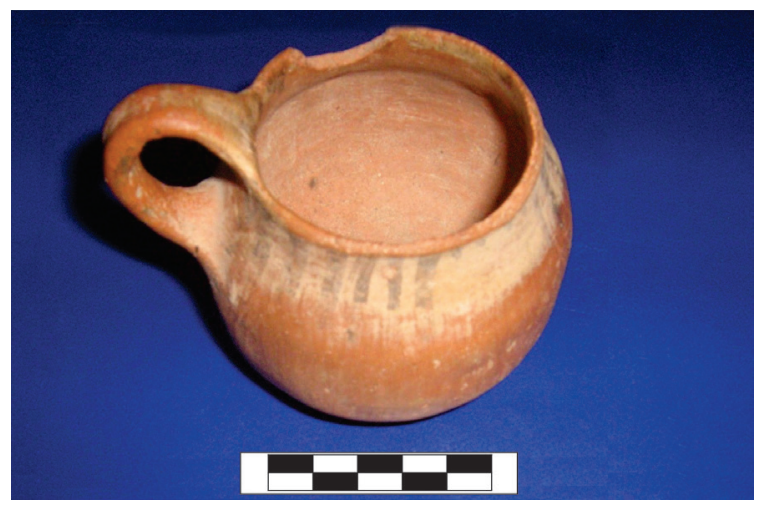

Figura 4. Jarrón Warpa Negro-Blanco sobre Ante.

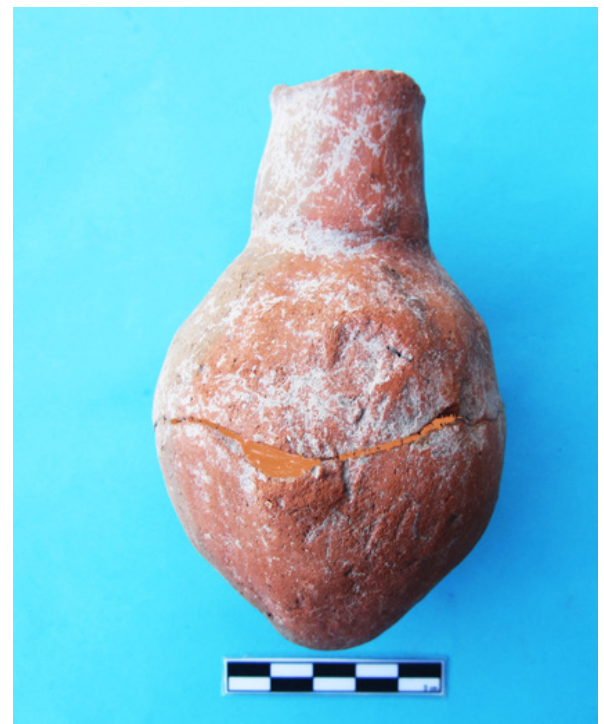

Figura 5. Botella de epoca Wari de Rudaqasa perteneciente a colección particular de la familia Palomino Mendoza.

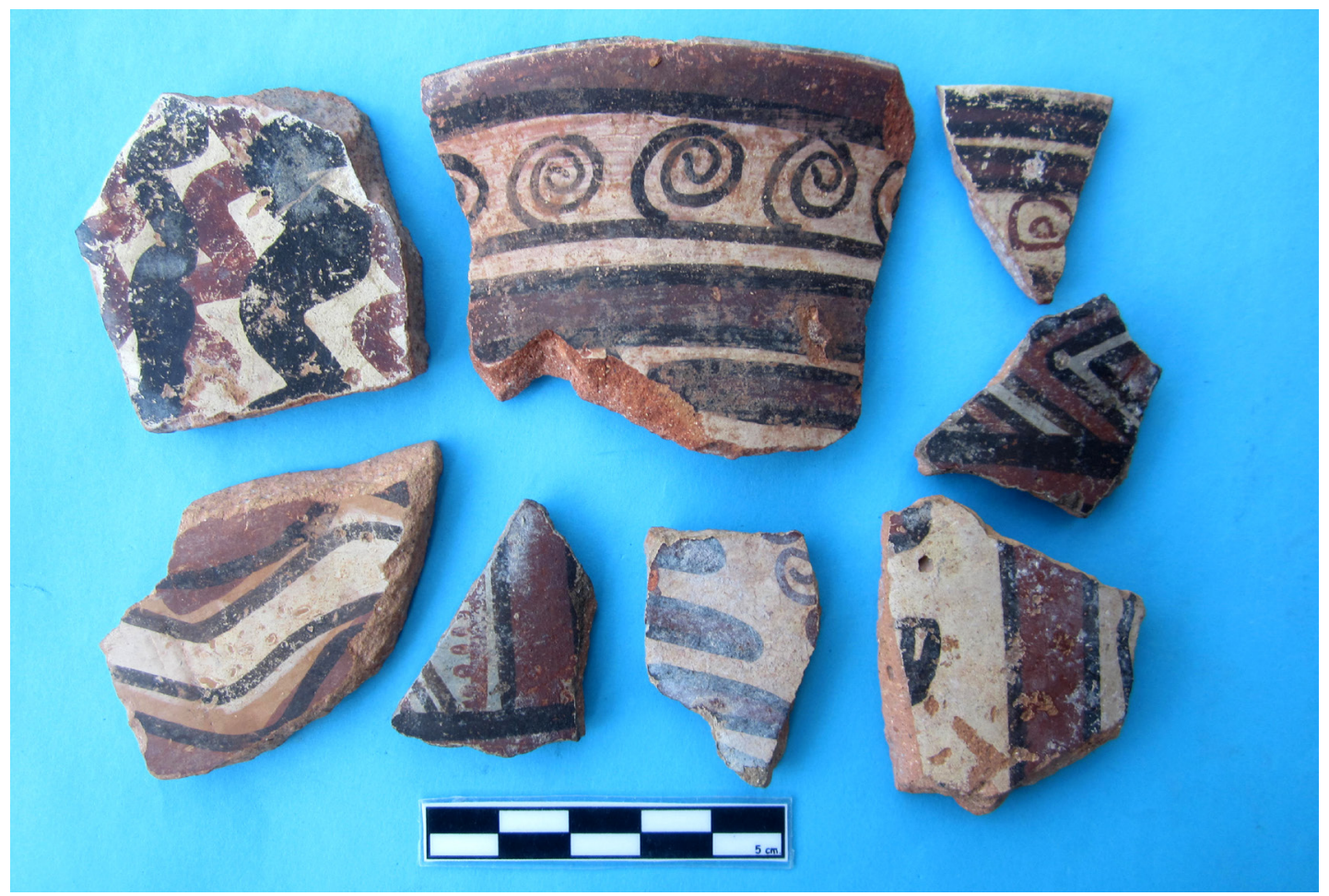

Figura 6. Detalle de Cucharas de época Warpa hallado durante la construcción de una vivienda en Rudaqasa, colección particular de la familia Mendoza Palomino. 
gro-Blanco sobre Ante, Warpa Negro sobre Blanco, Warpa Polícromo Zig-Zag, Cruz Pata Rojo- Marrón y el Warpa Tricolor. Además, una de los jarrones de colección particular, proveniente de Rudaqasa es el estilo Negro-Blanco sobre Ante, que apoya esta propuesta (figura 4). Por lo tanto, las evidencias de cerámica halladas y restos arquitectónicos asociados permiten comprender que se trata de un asentamiento importante que ha perdurado hasta el Horizonte Medio. Incluso la superposición estratigráfica develadas como parte del rescate arqueológico en Acuchimay por López (2014), hace vislumbrar una reocupación al igual que en Conchopata y Wari, las cuales en su amplitud es imposible de recuperar ya que tanto Acuchimay y Rudaqasa actualmente son parte de la ciudad moderna.

Después de los Warpa, durante el Horizonte Medio entre los siglos VI y VIII d. C., surge la cultura Wari, con su capital en el valle de Ayacucho; que fue reportado por primera vez por Cieza de León (2005, Capítulo LXXXVII) en el siglo XVI, denominado como Viñaca, con grandes y antiquísimos edificios preinca. Wari empieza con su expansión por gran mayor parte del territorio andino con el carácter de un estado teocrático y militarista que llegó dominar la mayor parte del territorio peruano llegando por el norte hasta Cajamarca, por el sur hasta el valle de Moquegua donde construyeron centros de poder del dominio religioso y control político (Williams \& Isla, 2002). Es una etapa de grandes cambios y movimiento poblacional que a los arqueólogos puso en debate y confrontaciones teóricas durante muchos años. En efecto el impacto en las culturas locales, a lo largo de los Andes Centrales parece que fue la organización social y la infraestructura económica, que permitió al pueblo ayacuchano extender sus límites territoriales (Isbell, 2001).

Al mismo tiempo durante esta etapa, también los Tiwanaku del altiplano boliviano llegaron a expandir su dominio comercial por el sur Andino, tomando la tradición iconográfica del Dios de los Báculos. Esta misma divinidad los Wari adoptan como uno de los símbolos de poder para su dominio y el avance territorial, cuyos indicios se muestra en las iconografías de arte religioso del estilo Conchopata, Robles Moqo y Viñaque. Al respecto Feldman (1998), enfatiza que el tema es complejo y se desconoce cómo ocurrió el paso de estas ideas de Tiwanaku a Wari, siendo hasta la fecha el área de Moquegua la que contiene pruebas significativas de una ocupación de ambas culturas juntas, caso único en comparación con otras zonas del Perú. Estos debates continúan en el interés arqueológico que dejamos fluir y pasamos a centrar nuestro interés en los poblados rurales alrededor de la ciudad Wari en la cuenca de Ayacucho.

La gran urbe Wari, ubicada en la planicie semiárida, durante su apogeo estaba circundada por numerosos asentamientos rurales y pequeñas ciudadelas. Entre los sitios conocidos y mejor estudiados hasta el momento al lado suroeste de la capital es 
Conchopata un poblado de alfareros del imperio Wari, dedicados a la producción de cerámica en mayor escala (Ochatoma, 2007). Asimismo, hay otros asentamientos de menor categoría como Tunasniyuq, Muyu Urqu, Aqu Wayqu, Nawinpukio, Rudaqasa y el mismo Acuchimay (Lumbreras, 1974), que fueron la base esencial para el desarrollo y el sustento de la capital. La población de este valle, como se ha mencionado desde los tiempos de la sociedad Warpa fue agrícola con un manejo del suelo y una tecnología adecuada en el campo hidráulico, que a la vez estaba complementada con la artesanía y la ganadería.

\section{ACUHIMAY UN PAISAJE SIMBÓLICO DESDE LAS ÉPOCAS PRE- HISPÁNICAS}

El paisaje arqueológico como un mundo exterior mediatizado por la experiencia subjetiva del hombre, son las dinámicas de las comunidades que trasforman el espacio físico mediante actividades diarias como las creencias y sistemas de valores que expresa lo material y lo imaginario (Prieto, 2011). Como parte de estos eventos socioculturales, la colina natural de Acuchimay ha sido modificado en un paisaje representativo no solo por su naturaleza física sino por el simbolismo vinculado a las montañas del valle de Ayacucho. Si bien su ubicación tiene una estrecha relación con las montañas sacralizadas del valle como Campanayuq, Picota, Qoriwillka, Santa Trinidad y Molinoyuq, siendo algunos de estos muy reverenciado hasta la actualidad en la hoya de Huamanga (Arroyo, 2006). Incluso desde el punto de vista espacial se encuentra rodeados por una serie de montañas mayores, entre los más conocidos Rasuwillka, Chukewaqra de Toccto, Cerro Lachoc de Vinchos y la cadena montañosa de Cerro Huacoto en las alturas Acosvinchos.

La veneración de montañas formaba parte central de la religión de los pueblos de Warpa en el valle de Ayacucho durante el periodo Intermedio Temprano, formando eventos públicos de gran importancia social y cultural para la comunidad local (Leoni, 2005). La importancia de Acumimay posiblemente adquirió un fuerte carácter sagrado, durante la época Warpa e incluso continuado durante el Horizonte Medio parte de la memoria hacia los ancestros. Incluso una de las representaciones iconográficas de cerámica procedentes de Conchopata manifiesta una comunidad amurallada en una cumbre de un cerro, que posiblemente se trataría de Acuchimay, como un asentamiento principal en los enclaves del valle de Ayacucho (Isbell, 2009). A este asentamiento se anexan otros sitios que tienen una ocupación desde las épocas tempranas, reocupado por los Warpa y Wari, como el caso de Waychaupampa, Ñawinpuquio, Yanama y Quicapata (Pérez, 2012, 2016), que configura con el rejuvenecimiento de Paisaje producto de la superposición de una cultura (Anschuetz et al., 2001). 

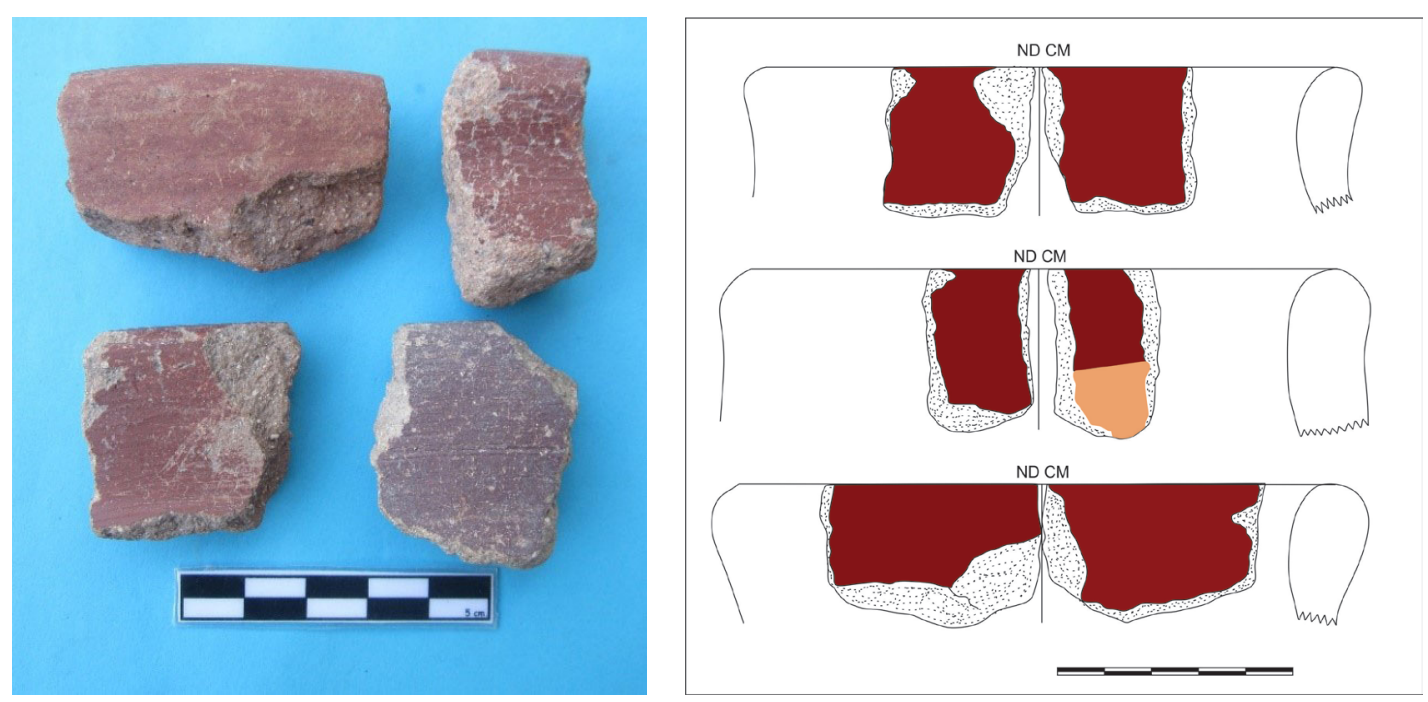

Figura 7. Detalle de cerámica estilo Kumunsenqa del periodo Intermedio Temprano, procedente de Acuchimay y Rudaqasa.
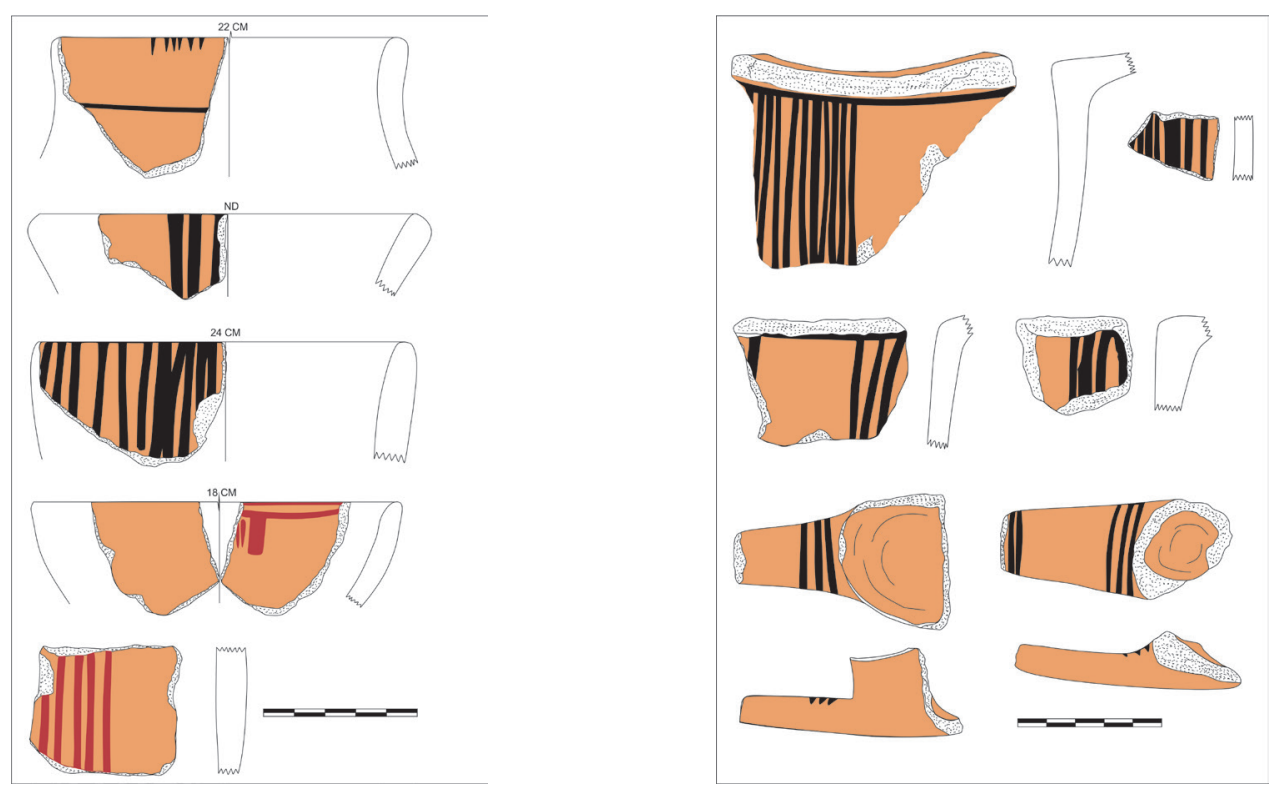

Figura 8. Detalle de cerámica estilo Warpa Negro sobre Ante y Warpa Rojo Sobre Ante, procedente de Acuchimay y Rudaqasa. 

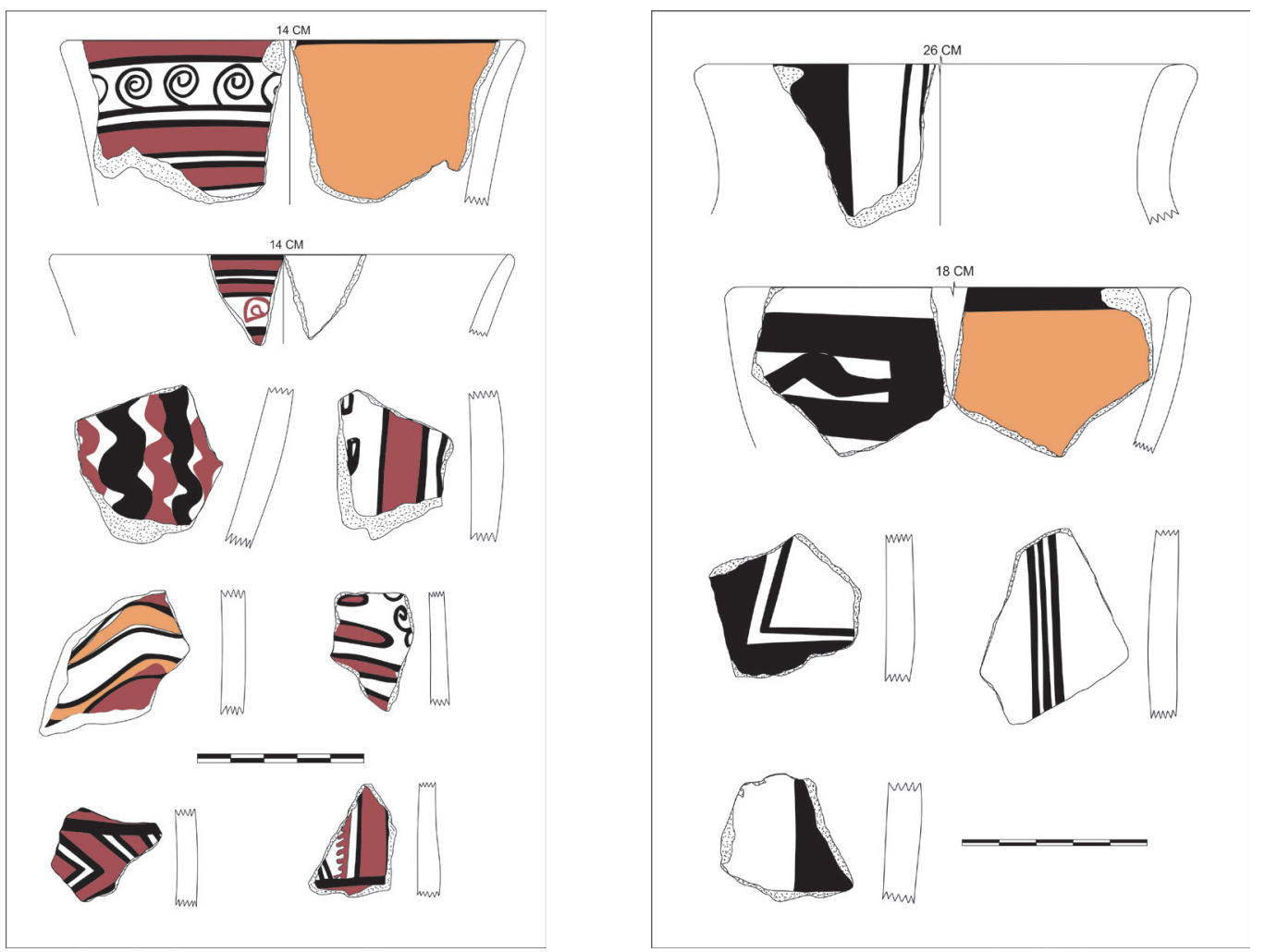

Figura 9. Detalle de cantaros y platos de cerámica Warpa estilos Negro sobre Blanco $y$ Warpa tricolor.

La materialidad recuperada en Acuchimay vislumbra una mirada más dinámica que permite comprender no solo el paisaje sagrado sino involucra al paisaje étnico en base a la cultura y símbolos materiales, que recrea una identidad sociocultural a la población actual en la cuenca de Ayacucho.

\section{CONCLUSIONES}

Acuchimay, uno de los aposentos prehispánicos de la cultura Warpa, fue modificado de manera dinámica desde el Periodo Intermedio Temprano (200 d. C. a 500 d. C.), con una reocupación durante el Horizonte Medio por los Wari (500 d. C. a 1000 d. C.), parte de la organización social y la infraestructura económica. Su ubicación actual dentro de la ciudad moderna ha llevado a la pérdida de la gran mayor parte del contexto cultural, al igual que los sitios Rudaqasa, Waychaupampa, Ñawinpuquio, Conchopata, Moyo Orqo, Tunasniyuq y Aqo Wayqo. Estos poblados menores cumplieron un rol muy fundamental en el valle de Ayacucho en las actividades artesanal, agrícola y ganadería. 
Desde el punto de vista de paisaje fue parte de una trasformación del espacio físico mediante creencias ideológicas y sistemas de valores con un simbolismo representativo hacia la veneración a las montañas locales. Esto implica que Acuchimay es un área ritual de una tradición muy remota, que perdura hasta la actualidad asociado a la medicina de la cosmovisión andina. Su ubicación céntrica articula a otras montañas sagradas de mayor prestigio, que cumplen diferentes roles dentro del mundo de los seres vivos y el medio natural. Por lo tanto, durante el transcurso de los años fue cambiando de manera progresiva con fases ocupacionales en diferentes épocas por los pobladores Warpa y Wari, trasladando al proceso de rejuvenecimiento del paisaje.

\section{REFERENCIAS BIBLIOGRÁFICAS}

Anschuetz, K., Wilshusen R. y Schieck, Ch. (2001). Una arqueología de los paisajes: perspectivas y tendencias. Journal of Archaelogical Reserch, 9 (2), 152-197.

Arroyo, S. (2006). Culto a los hermanos cristo. Sistema religioso andino y cristiano: redes y formas culturales del poder en los Andes (Tesis doctoral). Universidad Nacional Mayor de San Marcos, Lima, Perú.

Bennett, W. (1953). Excavations at Wari, Ayacucho, Perú. New Haven: Yale University. Publications in Anthropology 49.

Benavides, M. (1965). Estudio de la cerámica Decorada de Conchopata (Informe de grado de Bachiller). Universidad Nacional San Cristóbal de Huamanga. Ayacucho.

Benavides, M. (1976). Yacimiento arqueológico en Ayacucho. Ayacucho. Universidad Nacional de San Cristóbal de Huamanga, Departamentos Académicos de Ciencias Históricos Sociales.

Carhuas, H. (2020). Arqueología prehispánica y memoria histórica de la comunidad campesina San Martin de Yanapiruro e Ichubamba, Chiara-Ayacucho. Ayacucho.

Cieza de Leon, P. (2005). Crónica del Perú, el señorío de los incas. Caracas. Fundación Biblioteca Ayacucho.

Isbell, W. (2001). Huari: crecimiento y desarrollo de la capital imperial. Wari, arte precolombino peruano, 99-127. Fundación el Monte, Sevilla.

Isbell, W. (2009). Conchopata: Paisaje Urbano, Identidad, Producción artesanal en una Ciudad del Horizonte Medio. Conchopata Revista de arqueología, 2, 13-56. Universidad Nacional de San Cristóbal de Huamanga. Ayacucho.

Matos, R. (1981). Las culturas Regionales Tempranas. Historia del Perú antiguo. Tomo I, 353-524. Editorial Juan Mejía Baca, Lima, Perú. 
Ochatoma J. (2007). Alfareros del imperio Huari, Vida cotidiana y áreas de Actividad en Conchopata. Lima, Perú. Universidad Nacional San Cristóbal de Huamanga. VASPA SAC.

Ochatoma, J. y Cabrera, M. (2001). Pobladores rurales Huari, una visión desde Aqo Wayqo. Lima Perú. CANO asociados SAC.

Ochatoma, J. y Cabrera, M. (2010). Los Huarpas: caracterización y tipología cerámica. Investigación 2(18), 62-71. Universidad Nacional de San Cristóbal de Huamanga, Oficina general de investigación e innovación.

Ochatoma, J. y Cabrera, M. (2011). Iconografía y simbolismo en la cerámica de estilo Chakipampa-Huari. Investigación $N^{\circ}$ 02(19), 45-55. Universidad Nacional de San Cristóbal de Huamanga, oficina general de investigación e innovación.

Ochatoma, J. Cabrera, M. y Mancilla, C. (2015). El área sagrada de Wari, investigaciones arqueológicas en Vegachayuq Mogo. Ayacucho. Universidad Nacional de San Cristóbal de Huamanga, Vicerrectorado de investigación y oficina de investigación e innovación.

Peralta J. (1964). Tradiciones de Huamanga, volumen I. Ayacucho. Editor la miniatura.

Pérez, I., Cruz, N. y León, F. (2010). Arte rupestre en el valle de Huamanga, Ayacucho. Ayacucho, Perú.

Pérez, I. (2013). Asentamientos periféricos del centro urbano de Conchopata, Ayacucho. Arqueología y sociedad No 25, 143-168. Museo de Arqueología y Antropología de la Universidad Nacional Mayor de San Marcos.

Pérez, I. (2016). Secuencia cultural, previa a la cultura Huari en Ayacucho: Aportes y propuestas (Tesis de Magíster). Universidad Nacional mayor de San Marcos, facultad de ciencias sociales, unidad de posgrado.

Prieto, M. (2011). Los patrones de asentamiento: una herramienta metodológica para la reconstrucción del pasado. Boletín Antropológico 29(82),116-130. Universidad de Los Andes Venezuela.

Pulgar, J. (1996). Geografía del Perú, las ocho regiones naturales. Lima, Perú. PEISA.

Feldman, R. (1998). La ciudadela Wari de Cerro Baúl en Moquegua. Moquegua los primeros doce mil años. Una publicación del museo Contisuyo. Primera edición.

Leoni,B. J.(2005). La veneración de montañas en los andes preincaicos: el caso de Ñawinpukyo (Ayacucho, Perú) en el Periodo Intermedio Temprano. Chungara, Revista de Antropología Chilena, Volumen 37 (2), 151-164. 
López W. (2014). Excavaciones en Acuchimay, periferia oeste del Ñawimpuquio (Tesis de Licenciatura). Universidad Nacional de San Cristóbal de Huamanga, Ayacucho.

Lumbreras, L. G. (1959). Esquema Arqueológico de la sierra central del Perú. Revista del Museo Nacional, tomo XXVII, 63-116. Museo Nacional de Cultura, Lima.

Lumbreras, L. G. (1974). Las fundaciones de Huamanga. Hacia una prehistoria de Ayacucho. Lima, Perú. Homenaje al sesquicentenario de la batalla de Ayacucho. Primera edición.

Lumbreras, L. G. (2011). La estratificación arqueológica de Wari, según Bennett. Conchopata Revista Arqueológica N³, 49-70. Universidad Nacional de San Cristóbal de Huamanga. Ayacucho.

Menzel, D. (1968). La cultura Huari, las grandes civilizaciones del antiguo Perú, Tomo VI. Lima. Compañía de seguros y reaseguro peruano, Zuizo.S.A.

Tello, J. C. (2014). Cuaderno de investigaciones del archivo Tello $N^{\circ} 12$, expedición al Vilcamayo, 1942, primera parte: Sierra central y Cusco. Museo de Arqueología y Antropología Universidad Nacional Mayor de San Marcos.

Valdez, E. (2003). Sitios arqueológicos Huarpa en el valle de Huanta, Ayacucho. Revista Arqueológica Warpa Nº4,3-22. Huanta, Ayacucho, Perú.

Williams, P. y Isla, J. (2002). Investigaciones Arqueológicas en Cerro Baul. Un enclave Wari en el valle de Moquegua. Gaceta Arqueológica Andina N² 26, 87-120. Lima Perú. INDEA. 


\section{SOBRE EL AUTOR}

\section{Héctor Carhuas Tenorio}

Licenciado en Arqueología por la Universidad Nacional de San Cristóbal de Huamanga y candidato a magíster en Arqueología Andina por la universidad Nacional Mayor de San Marcos. Su línea de investigación está orientada en temas de Horizonte Medio y Horizonte Tardío, ha participado en diversos proyectos de investigaciones Arqueológicas y puesta en valor, destacando en las regiones de Ayacucho, Apurímac, Ica, Lima, Ancash, y Moquegua. Fue participe como ponente en eventos académicos de la Universidad Nacional de San Cristóbal de Huamanga, San Antonio de Abad del Cusco y San Luis Gonzaga de Ica. Asimismo, ha publicado diversos artículos académicos en revistas nacionales reconocidas y es autor del libro Arqueología Prehispánica y memoria histórica de la comunidad campesina San Martín de Yanapiruro e Ichubamba, Chiara-Ayacucho (2020). 